\title{
Role of Redcell Distribution Weight in Predicting Disease Severity, Mortality and Complication in Patients With Acute Pancreatitis
}

\author{
TAHTACI KARAALI REZAN ${ }^{1}$, TOPAL FIRDEVS', KARAKAYA ZEYNEP', \\ PAYZA UMUT ${ }^{1}$, KAVALCI CEMIL ${ }^{3}$, TOPAL FATIH ESAD ${ }^{1}$ \\ 1 Izmir Katip Çelebi University Atatürk Research and Training Hospital Emergency Department, Izmir/TURKEY
2 Izmir Katip Celebi University Atatürk Research and Training Hospital Gastroenterology Department, Izmir/TURKEY
3 Ankara Baskent University Hospital Emergency Department, Ankara/TURKEY
}

Corresponding author:

TAHTACI KARAALİ Rezan

Izmir Katip Celebi University Atatürk Research and Training Hospital Emergency Department, Izmir/TURKEY

+902322444444

rezantahtaci@hotmail.com

\section{ABSTRACT}

The goal of the present study was to investigate the significance of the Red Cell Distribution Width (RDW) in identifying the severity, mortality and complications of the disease at an early stage in patients with acute pancreatitis (AP).

343 patients with AP presented to the emergency department during one year were included in this retrospective study. Demographic, laboratory and imaging results were recorded. Bedside Index for Severity in AP (BISAP) score was calculated. The patients who developed pancreatitisrelated mortality were recorded.

The diagnostic powers of RDW values in the diagnosis of BISAP Score $(\geq 3)$, exitus, severe pancreatitis and pancreatitis with complication were analysed by means of Receiver Operating Characteristic Curve (ROC) analysis. $\mathrm{p}<0,05$ was considered statistically significant.

The mean age of the subjects was 59,7 $\pm 18,0$ years. Area under curve (AUC) in ROC analysis conducted for RDW in patients with BISAP score $\geq 3$ was 0,649 $(95 \% \mathrm{Cl} 0,576-0,722)$ and $\mathrm{p}<0,001$. For developed complications, AUC for RDW was 0,558 (95\% Cl 0,454-0,662) and $\mathrm{p}$ was 0,243 . For $\mathrm{RDW}<14,4$ cut-off value; the sensitivity was $66,25 \%$, specificity was $71,48 \%$ in the prediction of BISAP $\geq 3$, sensitivity was $87,5 \%$, specificity was $65,14 \%$ in the prediction of mortality, sensitivity was $72,73 \%$, specificity was $71,12 \%$ in the prediction of severe AP.

RDW is as significant as the BISAP score in predicting the severity and mortality of pancreatitis in the patients with acute pancreatitis in the emergency
department(ED). But it cannot predict the complications in AP.

Keywords: Emergency, acute pancreatitis, mortality, severity, complications, redcell distribution weight.

\section{INTRODUCTION}

Acute pancreatitis(AP) is acute inflammatory disease of the pancreatic gland, which usually develops in connection with gallstones or alcohol consumption and progresses with local or systematic symptoms. The disease may begin with mild symptoms, such as abdominal pain, nausea and vomiting and if not treated, it can lead to the development of multiple organ dysfunction syndrome, common metabolic disorder or sepsis (1-3). The prognosis of the disease depends on the severity of the disease.

According to the Atlanta 2012 classification, it is divided into three types - moderate acute pancreatitis (MAP), modarately severe acute pancreatitis (MSAP) and severe acute pancreatitis (SAP) $(4,5)$. MSAP makes up almost $80 \%$ of all pancreatitis patients and its mortality is around $5 \%(2,6,7)$. While mortality in MAP and MSAP was $1-3 \%$, this rate rises up to 20 $25 \%$ in SAP $(6,8)$. It is important to get an early risk assessment within the first 24-48 hours for the patients with AP in the emergency department.

Many studies suggest the use of some scoring systems and laboratuary tests to predict the severity of acute pancreatitis, including; Balthazar Computed tomography severity index (9), Bedside Index for Se- verity in Acute Pancreatitis score (BISAP) $(7,10,11), C$ reactive protein $(\mathrm{CRP})(12,13)$, blood urea nitrogen(BUN), D- Dimer, procalcitonin levels $(11,14)$. Also, the Ranson and Acute Physiology, Age, Chronic Health Evaluation II (APACE II) systems were used for this purpose $(10,15,16)$. However, they are difficult to figure out by the bedside since they are clumsy and complicated.

The BISAP score is a method of scoring calculated based on 5 variables within the first 24 hours of admission to the emergency room. These variables are BUN> $25 \mathrm{mg} / \mathrm{dl}$, state of consciousness, development of Systemic Inflammatory Response Syndrome(SIRS) symptoms, age being older than 60, presence of pleural effusion; each parameter is counted as 1 point and the score is calculated accordingly (11). BISAP has been found as reliable as other scores, in the evaluation of patients with AP $(7,8,10,15)$.

Red Cell Distribution Weight (RDW) is an easy, routine and inexpensive test, as a parameter that can be obtained through a complete blood count. RDW is the quantitative variation in the size of circulating red blood cells (RBCs). The rise of RDW indicates that the erythrocytes are in the circulation, in different volumes and different ways $(2,14,17)$. RDW also increases in coronary artery disease, stroke, kidney failure and severe infections (18).

Pathophysiological mechanisms between mortality and high RDW are not clear in AP. Inflammation can help to explain them. Inflammation affects the bone marrow and iron metabolism (19). Fatality in acute pancreatitis occurs either due to sepsis which already exists since the beginning 
or develops following a cytokine storm developing in the early stage $(3,8,14)$. For all these reasons, RDW gained popularity in predicting the severity and mortality especially in AP patients $(1,2,18,20)$. The early diagnosis of AP patients in the emergency department and quick aggressive treatment reduces the mortality. Therefore, simple, easily accessible and affordable markers are needed, which allow investigation on the bedside whether the disease will progress severely and enable the prediction of the progress of the complication and the mortality, without any time restriction. In this study, we aimed to investigate the role of RDW in predicting the severity of the disease and mortality in the cases of AP and in the prediction of pseudocyst, pancreatic necrosis and pancreatic abscess which are the complications of pancreatitis.

\section{MATERIALS AND METHODS}

\section{Patient selection}

This retrospective study was conducted between January 1st, 2017 and December 31st, 2017 at Ataturk Training and Research Hospital. An average of 800 patients are admitted to the emergency department of our hospital in 24 hours. It is a centre that receives referrals from other hospitals as it is a 3rd Level hospital which has a gastroenterology clinic with ERCP, MRCP and EUS facilities. The patients who have visited our emergency department and have been diagnosed with AP according to ICD-10 codes were reviewed. 343 patients aged above 18 years old, for whom sufficient data was obtained in their files, were included in the study. The patients under 18 years of age, expecting (pregnant) women, the patients receiving radiotherapy-chemotherapy, the patients with severe renal failure, those with anaemia (including those receiving B12, iron therapy) and immunodeficiency patients were excluded from the study.

\section{Data collection}

Based on the laboratory records obtained through the system, White blood cell (WBC), blood urea nitrogen (BUN), amylase, lipase, aspartate amino transferase (AST), alanine amino transferase (ALT), red blood cell distribution weight (RDW) values were recorded in the form created. Imaging methods used and the results achieved were examined. Endoscopic retrograde cholangiography (ERCP), computerized tomography (CT), endoscopic ultrasonography (EUS) and magnetic resonance cholangiography (MRCP) findings were recorded. (BISAP) scores of the patients were calculated using the data obtained, and according to 2012 Atlanta classification; the patients with $\mathrm{BISAP}<3$, no organ failure or local complications were classified as moderate acute pancreatitis (MAP) (Group 1); the patients with $\mathrm{BISAP} \geq 3$, no permanent organ failure but detected with pseudocyst, pancreatic necrosis and pancreatic abscess which are the complications of pancreatitis, were classified as moderately severe acute pancreatitis (MSAP) (Group 2); the patients with $\mathrm{BISAP} \geq 3$ and at least one persistent organ failure were classified as severe acute pancreatitis (SAP) (Group 3), and also the patients detected with at least one of the findings of pancreatic abscess, pseudocyst and necrosis were classified as pancreatitis with complications (Group 4). The patients developing pancreatitis-related mortality were recorded.

\section{Statistical method}

Statistical analysis of the data was performed using IBM Statistics Version 24.00 program. Distribution of continuous data was examined with Kolmogorov Smirnov test. Mann Whitney U statistical analysis was used to compare the continuous data between the two groups. The diagnostic powers of RDW values in the diagnosis of Score $(\geq 3)$, exitus, severe pancreatitis and pancreatitis with complication were analysed by means of Receiver Operating Characteristic Curve (ROC) analysis. $\mathrm{p}<0,05$ was considered statistically significant.

\section{RESULTS}

The average age of 343 patients were included in the study is $59,7 \pm 18,0$ years, 153 of whom were male $(45,6 \%)$ and 190 were female $(53,4 \%)$. Looking at the severity of the disease; $19.2 \%$ of patients were found to be SAP, $69.68 \%$ were MAP and MSAP, $4.7 \%$ were exitus. The rate of patients developing at least one of the complications was found $11.1 \%$. The score value of $23,3 \%$ patients was found 3 and above, considering the distribution according to the BISAP Score.

The most common etiological cause was found to be the gallstones by $52,5 \%$. Others included 39,4\% idiopathic, 5,2\% cancer, $2.0 \%$ post ERCP, $1.2 \%$ alcohol. Looking at the proportion of the imaging methods used; $87,8 \%$ EUS, $36,2 \%$ CT, $28,9 \%$ ERCP and $6,7 \%$ MRCP were performed.

Examining the relationship of the laboratory results obtained, with BISAP score, survival/recovery, severe pancreatitis and complication; ALT, AST, BUN, CRP and RDW values of the patients with a BISAP score of $\geq 3$ were found significantly higher

Table 1. Relationship of laboratory values with BISAP score.

\begin{tabular}{llll}
\hline & BISAP SCORE & p \\
\hline & $<3$ & $>3$ & \\
\hline Alanine Aminotransferase & $87,5(196,25)$ & Median (IQR) & 0,000 \\
\hline Amylase & $706(1356)$ & $322(367)$ & 0,055 \\
\hline Aspartate Aminotransferase & $94(163,75)$ & $1011,5(1503,5)$ & 0,000 \\
\hline Blood Urea Nitrogen & $14(8)$ & $320(401)$ & 0,000 \\
\hline C-Reaktive Protein & $1,21(4,665)$ & $21(15,75)$ & 0,015 \\
\hline Lipase & $1794(3914,5)$ & $2,51(7,6975)$ & 0,103 \\
\hline Redcell Distributionon Weight & $13,8(1,5)$ & $2316,5(4687,5)$ & 0,000 \\
\hline White Blood Cell & $10,98(5,7)$ & $14,95(2,175)$ & 0,005 \\
\hline
\end{tabular}


Table 2. The relationship of laboratory values with mortality

\begin{tabular}{llll}
\hline & MORTALITY & p \\
\hline & live & Ex & \\
\hline Alanine Aminotransferase & Median (IQR) & Median (IQR) & 0,140 \\
\hline Amylase & $111(263)$ & $36,5(268,5)$ & 0,643 \\
\hline Aspartate Aminotransferase & $117(211,5)$ & $609,5(1374,75)$ & 0,599 \\
\hline Blood Urea Nitrogen & $15(9)$ & $88(317)$ & 0,001 \\
\hline C-Reaktive Protein & $1,41(5,0825)$ & $23,5(13,5)$ & 0,127 \\
\hline Lipase & $2072(3992)$ & $2,57(10,2175)$ & 0,376 \\
\hline Redcell Distributionon Weight & $13,9(1,725)$ & $1397(2120,75)$ & 0,001 \\
\hline White Blood Cell & $11,2(5,6775)$ & $15,25(1,25)$ & 0,506 \\
\hline
\end{tabular}

Table 3. The relationship of laboratory values with severity of disease.

\begin{tabular}{llll}
\hline & SEVERE PANCREATITIS & $\mathbf{p}$ \\
\hline & NO & YES & \\
\hline Alanine Aminotransferase & $88(193,75)$ & Median (IQR) & 0,000 \\
\hline Amylase & $698(1379,25)$ & $374(364)$ & 0,006 \\
\hline Aspartate Aminotransferase & $97(161,75)$ & $1114(1748,5)$ & 0,000 \\
\hline Blood Urea Nitrogen & $15(8,75)$ & $381,5(427,5)$ & 0,000 \\
\hline C-Reaktive Protein & $1,4(5,4475)$ & $19,5(15)$ & 0,387 \\
\hline Lipase & $1791(3668)$ & $1,99(4,8225)$ & 0,013 \\
\hline Redcell Distributionon Weight & $13,8(1,5)$ & $2502,5(4762,5)$ & 0,000 \\
\hline White Blood Cell & $11,04(5,845)$ & $15,05(2,05)$ & 0,047 \\
\hline
\end{tabular}

Table 4. The relationship of laboratory values with complications.

\begin{tabular}{llll}
\hline & COMPLICATION & p \\
\hline & NO & YES & \\
\hline Alanine Aminotransferase & Median (IQR) & Median (IQR) & 0,012 \\
\hline Amylase & $788(1380,5)$ & $62(106,5)$ & 0,230 \\
\hline Aspartate Aminotransferase & $121,5(217)$ & $802(1161)$ & 0,152 \\
\hline Blood Urea Nitrogen & $16(10,5)$ & $69(208)$ & 0,266 \\
\hline C-Reaktive Protein & $1,43(4,815)$ & $15,5(8,5)$ & 0,063 \\
\hline Lipase & $2155(3912,5)$ & $2,74(14,96)$ & 0,100 \\
\hline Redcell Distributionon Weight & $14(1,8)$ & $964(4216)$ & 0,243 \\
\hline White Blood Cell & $11,06(6,2)$ & $14,05(2,75)$ & 0,134 \\
\hline
\end{tabular}

than the values of cases whose scores were less than " 3 " $(\mathrm{p}<0,05)$ (table 1$)$. The BUN and RDW values of the exitus cases were found significantly higher than the surviving cases $(\mathrm{p}<0,05)$ (table 2$)$. ALT, amylase, AST, BUN, lipase, RDW, WBC, and values of the cases detected with severe pancreatitis were found significantly higher than the cases with no severe pancreatitis $(\mathrm{p}<0.05)$ (table 3 ). The parameters above were not statistically significant in MAP and MSAP. Laboratory values in the patients classified as having complications were not found statistically significant as compared to the other groups (table 4). In terms of other variables, no statistically significant difference was found between the groups ( $\mathrm{p}>0$, $05)$. The result of ROC analysis conducted for the power of RDW in predicting the mortality showed: RDW AUC 0,754 (95\% Cl 0,670-0,838), $\mathrm{p}<0.001$ (Graphic1). For RDW $>14.4$ cut-off value of RDW values; sensitivity in predicting mortality was $87.5 \%$ and specificity $65.14 \%$.

\section{DISCUSSION}

Acute pancreatitis is an inflammatory disease of the pancreas, which can lead even to death if left untreated. The factors causing inflammation are mostly the gallstones and use of alcohol. Looking at the previous studies, the rate of developing acute pancreatitis due to gallstone was found between $40-63 \%(2,3,6,16)$. In our study, we found the rate of developing acute pancreatitis due to gallstone as $52,5 \%$, in com- 
pliance with the literature. When examined according to the gender of the patients, $55,84 \%$ of the patients in our study were female, with an average age of 59,7.

Mortality in acute pancreatitis is associated with multiple organ failure and septic complications. The development and progression of multiple organ failure is related to the inflammatory process. The severity of inflammation affects the course and mortality of the disease. Based on this fact, two periods were defined, at which the mortality peaks in the course of AP. The first is the early period which may lead to multiple organ failure and SIRS that develops in the first week and the second is the late period during which persistent organ dysfunction is observed, accompanied by infection and sepsis that develop between 1-3 weeks $(17,21)$. Early detection of the severity of the disease and initiation of proper treatment reduces the late period complications and mortality. A number of Scoring systems have been developed to predict the severity and mortality in AP. BISAP score, which is one of them, was developed for the first time by $\mathrm{Wu}$ et al, in an attempt to predict the severity of disease in patients with AP. In this study, the reliability of the score was compared to APACHE II score value calculated in patients with AP (11). 12 articles were examined in the review study conducted by Subash et al. and it was found that BISAP displayed a good performance in the prediction of SAP and BISAP score was 3 and above particularly in patients with a severe pancreatitis and persistent organ failure lasting longer than 48 hours (7). Ting $\mathrm{Z}$ et al., found out in their study that BISAP $\geq 3$ value for the patients with RANSON score $\geq 3$, APACHE II score $\geq 8$ had a correlation with the severity of AP (2). In our study, the diagnosis of SAP was statistically significant in patients with BISAP $\geq 3$. RDW is a parameter that shows the distribution width of the circulating erythrocytes. Its level increases during inflammatory periods. In the RDW studies conducted by Kilic M. O. et al., and Ucar Karabulut K. et al., it was discovered that RDW has predicted the severity of AP $(16,20)$. In the study performed by Thing Z. et al., on the patients diagnosed with AP, RDW has a correlation with BISAP in predicting the severity of AP (2). In our study, RDW 14,92 $\pm 1,67$ value for patients with BISAP Score $\geq 3$ was found to be statistically significant $(\mathrm{p}<0,0005)$.

Studies with RDW have shown that it is related not only to the severity but also to the mortality of the disease. Especially, it was revealed that RDW is related with the mortality in patients with cardiac dis-

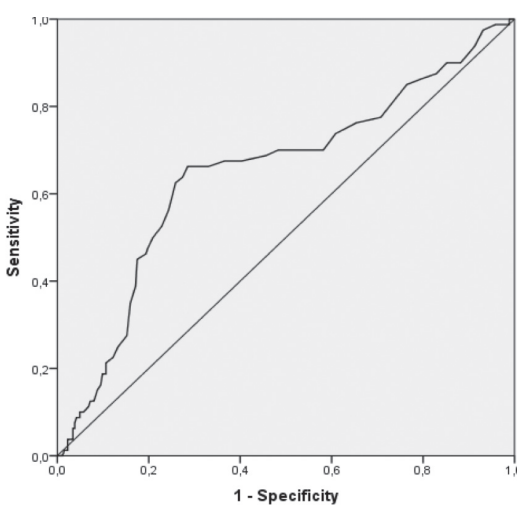

Graphic 1. ROC analyse of comparasion BISAP score with RDW

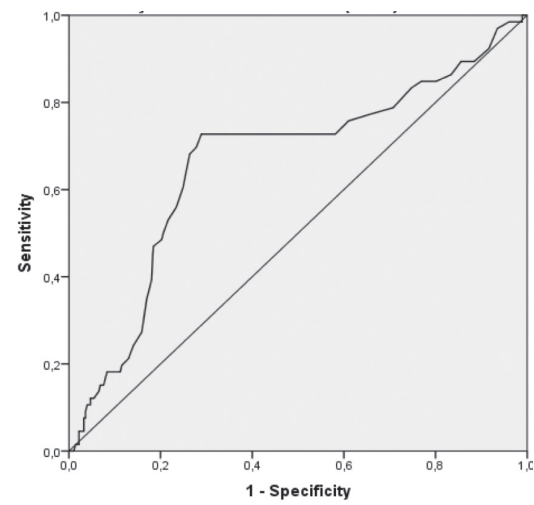

Graphic 3. ROC analyse of RDW with severity of disease.

eases, malignancies, infections, renal diseases, pulmonary diseases and the patients with cerebro vasculer disease in particular $(17,22)$. In the study conducted by Jo et al., where they investigated the relationship of RDW with mortality in patients with severe sepsis and septic shock, RDW level was found out to be considerably high in the exitus (22). RDW is nowadays used to predict the mortality in AP which is an inflammatory disease. This is because mortality in AP is associated with multiple organ failure and septic complications. Ina study by Payman M. et al., elevated RDW was found to be associated with mortality in patients diagnosed with AP and that 14.55 as the cutoff value of RDW had $80 \%$ sensitivity, $85 \%$ specificity (1). In a study by Wenzheng Z. et al., RDW was compared with albumin and creatinine in determining the mortality in AP. The values of RDW at and above 14.45 was found to predict the mortality with a sensitivity of $88,9 \%$ (3). In the review study of Hemant G. et al., 7 retrospective studies were examined and it was noted that RDWwas significantly higher in the deceased patients than in the survivors. Furthermore, in 4 of these studies, RDW was found to be an independent risk factor as an indicator

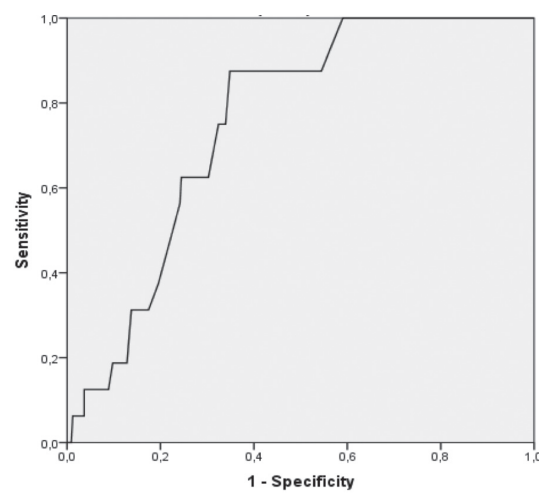

Graphic 2. ROC analyse of RDW with mortality.

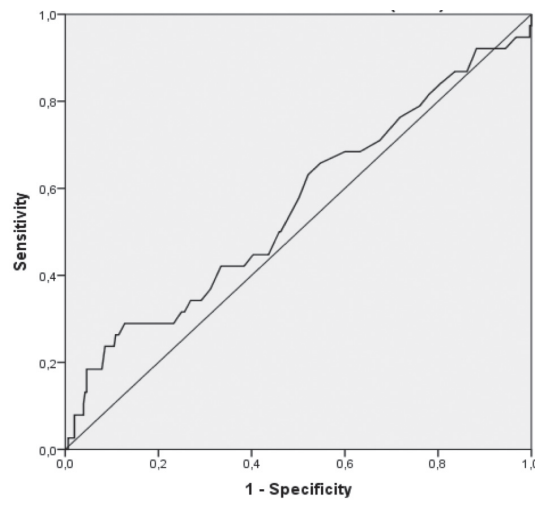

Graphic 4. ROC analyse of RDW with complications.

of mortality (14). In a study performed by Senol et al, 14,8 value of RDW was found significant in predicting the mortality in patients with AP. And also, when compared toWBC, platelet, BUN, calcium and albumin, RDW was found as an independent prognostic factor which predicts the mortality (17). In the study by Yao et al., they compared the RDW values of the patients with acute pancreatitis to those of the normal population. They divided the pancreatitis patients into two groups as SAP and MAP and RDW wasfound significantly high in SAP patients and the exitus (18). In our study, RDW $>14,4$ cut-off value had $87,5 \%$ sensitivity, $65,14 \%$ specificity in predicting the mortality. According to these results, our study supported the fact that RDW is related with the severity and mortality of the disease.

When the literature is reviewed, there is no study investigating whether RDW also predicts the complication, as well. Development of complication affects the mortality and rate of morbidity in acute pancreatitis. In the study conducted by Biberoglu et al., it was found out that two prognostic factors have been used in calculation of CT severity index and whether one of them 
is necrosis and the amount of necrosis is a factor affecting the mortality directly (9). Therefore, in our study, we classified the patients detected with abscess, necrosis and pseudocyst as a separate group and looked into the relationship with RDW. In our study, no statistical relationship was found between the RDW value measured in the blood sample taken from the patient at emergency department and development of complication. No significant result was obtained in the ROC analysis performed with RDW value measured for patients who have developed complications.
Although we cannot fully explain why RDW cannot predict the complication in cases with complications, this may be due to the fact that complications develop later in the disease process, e.g. 1 and 3 weeks later, and the patient does not produce any systemic warning as he/she is under treatment and therefore does not affect the hematopoietic system. Our study is the first to investigate the relationship between the cases developing complications in pancreatitis and RDW in the literature. However, it needs further and comprehensive studies on this subject.
In conclusion, RDW value in patients with acute pancreatitis is as valuable as the BISAP score in predicting the severity and mortality of pancreatitis in the emergency department. It is advantageous as opposed to BISAP, in that it can be treated on the bedside and no additional parameters are needed. Moreover, it can be obtained through affordable and routine blood count. But it cannot predict the complications in AP.

\section{REFERENCES}

1. Payman M, Kavous S.N, Mohammadhossein S, Mahboub P, Akbar F, Farzad R. Red blood cell distribution width: a determinant of hospital mortality in pancreatitis. JEPT 2018; 4(1), 2018: 34-38.

2. Ting Z, Hua L, Dongyan W, Pengfei Z, Changfeng G, Feng et al. Predicting the severity of acute pancreatitis with red cell distribution width at early admission stage. Shock.2018 May;49(5):551-555.

3. Wenzheng Z, Jiang H, Bihui Y, Xvsheng Y, Lei S, Ting Y, et al. Evaluation of Early Prognostic Factors of Mortality in Patients with Acute Pancreatitis: A Retrospective Study. Gastroenterol Res Pract 2017; 2017:1-6

4. Banks PA, Bollen TL, Dervenis C, Gooszen HG, Johnson CD, Sarr MG, et al. Classification of acute pancreatitis--2012: revision of the Atlanta classification and definitions by international consensus. Gut 2013 Jan;62(1):102-11.

5. Sarr MG . 2012 revision of the Atlanta classification of acute pancreatitis. Pol Arch Med Wewn. 2013;123(3):118-24.

6. Jiang-Feng Y, Yu-Xin Z, Jian J, Wei W. Building and verifying a severity prediction model of acute pancreatitis (AP) based on BISAP, MEWS and routine test indexes. Clin Res Hepatol Gastroenterol. 2017 Oct;41(5):585-591.

7. Subhash C, Arvind M, Reena B, Dipti A, Adrian H. The Bedside Index for Severity in Acute Pancreatitis: a systematic review of prospective studies to determine predictive performance. J Community Hosp Intern Med Perspect 2017; 7(4): $208-213$.

8. Vasudevan S, Goswami P, Sonika U, Thakur B, Sreenivas V, Saraya A.. Comparison of Various Scoring Systems and Biochemical Markers in Predicting the Outcome in Acute Pancreatitis. Pancreas. 2018 Jan;47(1):65-71.

9. Biberoğlu S, Koyuncu M, Öztürk D, Toksöz A.C, ÇakmakcıE, Çakmak F, et al. Role of Computed Tomography Severity Index in Acute Diagnosis and Follow-up of Acute Pancreatitis. J Clin Anal Med 2017;8(2): 106-110.

10. Gao W, Yang HX, Ma CE. The value of BISAP score for predicting mortality and severity in acute pancreatitis. A sistematic review and meta-anlysis. PLoS One. 2015 Jun 19;10(6): e0130412.

11. Wu BU, Johannes RS, Sun X, Tabak Y, Conwell DL, Banks PA. The early prediction of mortality in acute pancreatitis: a large population-based study. Gut. 2008 Dec;57(12):1698-703

12. Vinish DB, Abishek V, Sujatha K, Arulprakash S, Solomon R, Ganesh P.Role of bedside pancreatic scores and C-reactive protein in predicting pancreatic fluid collections and necrosis. Indian J Gastroenterol. 2017 Jan;36(1):43-49.

13. Zheng J, Zhang J, Gao J. Role of bedside pancreatic scores and C-reactive protein in predicting pancreatic fluid collections and necrosis. Zhonghua Yi Xue Za Zhi. 2015 Mar 31;95(12):925-8.

14. Goyal H, Awad H, Hu ZD. Prognostic value of admission red blood cell distribution width in acute pancreatitis: a systematic review. Ann Transl Med. 2017 Sep;5(17):342.

15. Yang L, Liu J, Xing Y, Du L, Chen J, Liu X, et al.Comparison of BISAP, Ranson, MCTSI, and APACHE II in predicting severity and prognoses of hyperlipidemic acute pancreatitis in chinese atients. Gastroenterol Res Pract. 2016;2016:1834256

16. Kılıç MÖ, Çelik C, Yüksel C, Yıldız BD, Tez M. Correlation between Ranson score and red cell distribution width in acute pancreatitis. Ulus Travma Acil Cerrahi Derg. 2017 Mar;23(2):112-116.

17. Şenol K, Saylam B, Kocaay F, Tez M.Red cell distribution width as a predictor of mortality in acute pancreatitis. Am J Emerg Med. 2013 Apr;31(4):687-9.

18. Yao J, Lv G.Association between red cell distribution width and acute pancreatitis: a cross-sectional study. BMJ Open. 2014 Aug 5;4(8):e004721.

19. Cakal B, Akoz AG, Ustundag Y, Yalinkilic M, Ulker A, Ankarali H.Red cell distribution width for assessment of activity of inflammatory bowel disease. Dig Dis Sci. 2009 Apr;54(4):842-7.

20. Uçar Karabulut K, Narcı H, Uçar Y, Uyar M.Association between Red blood cell distribution width and acute pancreatitis. Med Sci Monit. 2014 Nov 27;20:2448-52.

21. Harper S.J.F and Cheslyn-Curtis S. Acute pancreatitis. Ann Clin Biochem 2011; 48: 23-37.

22. Jo YH, Kim K, Lee JH, Kang C, Kim T, Park HM, et al. Red cell distribution width is a prognostic factor in severe sepsis and septic shock. Am J Emerg Med. 2013 Mar;31(3):545-8 